\title{
Angle-scanned photoemission: Fermi surface mapping and structural determination
}

\author{
P. Aebi a,*, R. Fasel a, D. Naumović a, J. Hayoz a , Th. Pillo a , M. Bovet a \\ R.G. Agostino ${ }^{\text {a }}$, L. Patthey ${ }^{\text {a }}$, L. Schlapbach ${ }^{\text {a }}$, F.P. Gil ${ }^{\text {b }}$, H. Berger ${ }^{\text {c }}$, T.J. Kreutz ${ }^{\text {d }}$, \\ J. Osterwalder ${ }^{\mathrm{d}}$ \\ ${ }^{a}$ Institut de Physique, Université de Fribourg, Pérolles, CH-1700 Fribourg, Switzerland \\ ${ }^{\mathrm{b}}$ Departmento de Fisica, Universidade de Coimbra, P-3000 Coimbra, Portugal \\ ${ }^{\mathrm{c}}$ Institut de Physique Appliquée, EPFL, CH-1015 Lausanne, Switzerland \\ ${ }^{d}$ Physik-Institut, Universität Zürich-Irchel, Winterthurerstr. 190, CH-8057 Zürich, Switzerland
}

Received 18 August 1997; accepted for publication 2 October 1997

\section{Abstract}

A brief survey of the angle-scanned photoemission technique is given. It incorporates two complementary methods in one:

(1) Mapping of X-ray excited photoelectron intensities over virtually the complete hemisphere above the sample surface results in extended data sets where important surface-geometrical structure information is extracted and even "fingerprinting" is possible. This method is known as the very powerful angle-scanned X-ray photoelectron diffraction.

(2) Mapping ultraviolet-excited photoelectron intensities as a function of emission angles gives the possibility to do band mapping as well as to study the Fermi surface of single crystals very directly. Therefore, by switching between X-rays and ultravioletphotons, it is possible to study the geometrical and electronic structure within the same experiment. (C) 1998 Elsevier Science B.V. All rights reserved.

Keywords: Angle-scanned photoemission; Band mapping; Fermi surface mapping; Photoelectron diffraction; Surface structure

\section{Introduction}

Photoemission has a long-standing tradition in surface analysis. X-ray photoelectron spectroscopy (XPS) is used to study the chemical composition of surfaces. In the ultraviolet (UV) regime, using UV photoelectron spectroscopy, typically with a He discharge lamp, the acquisition of energy distribution curves (EDCs) gives information on the electronic structure near the surface.

* Corresponding author. Fax: (+41) 2630097 47; e-mail: philippadrian.Aebi@unifr.ch
On single crystals, using angle-resolved photoemission, it is possible to study the atomic structure, i.e. atomic positions, using the X-ray photoelectron diffraction (XPD) technique as well as the electronic structure, i.e. the band structure, in the UV regime. The combination of both excitation sources in the same experiment is very valuable since electronic and atomic degrees of freedom are interdependent. Traditionally angle-resolved experiments have been performed for rather few angles preferentially along high symmetry directions where complete EDCs were collected. Here, we report on experiments using extensive angle- 


\section{Angle-scanned photoemission}
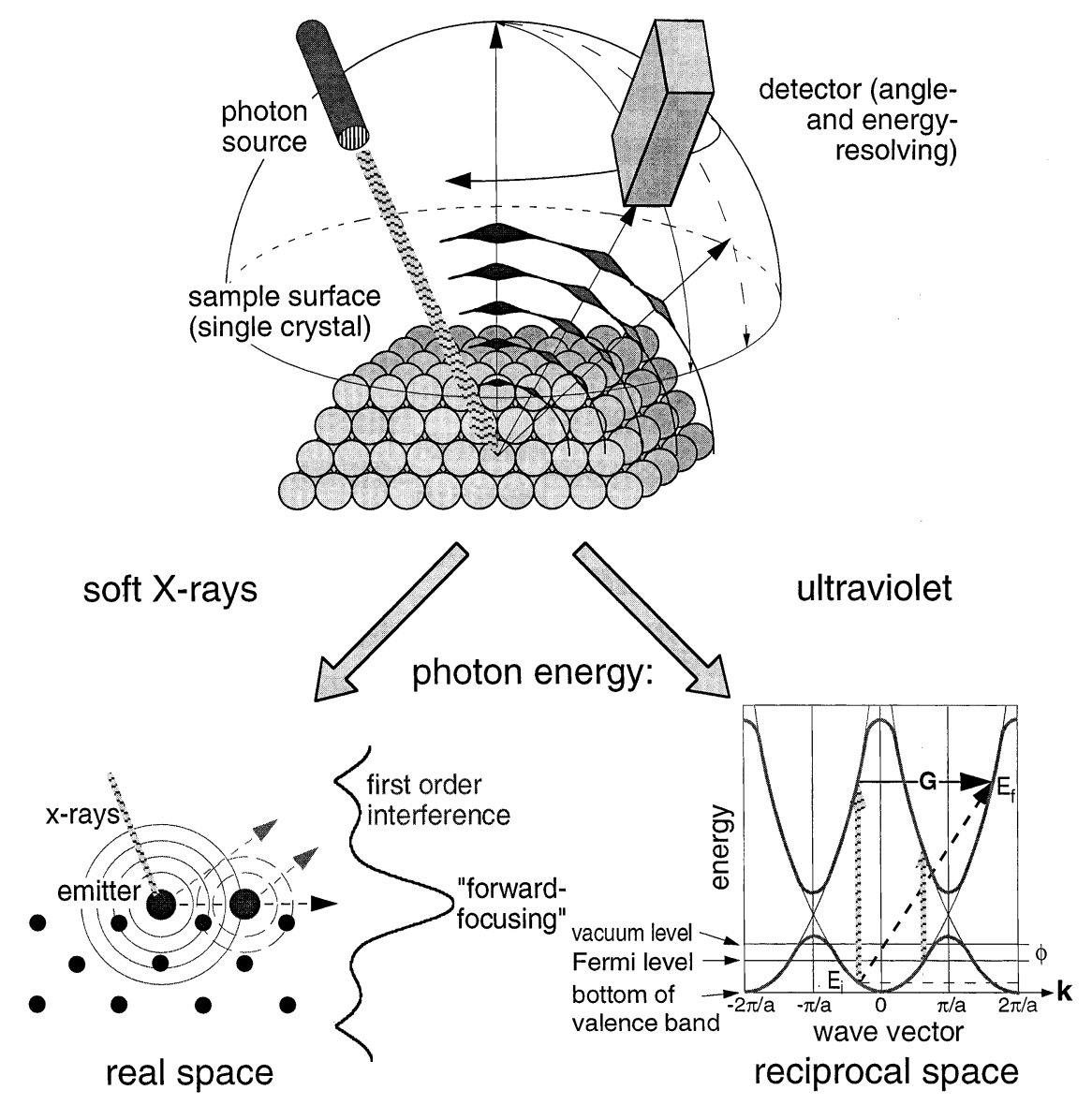

Fig. 1. General principle of angle-scanned photoemission. Photoelectron intensities are collected over much of the hemisphere above the sample surface. Switching between soft X-rays and UV photons either real-space or reciprocal-space information can be gained (see text)

scanning, covering much of the hemisphere above the sample surface using both X-rays and UV photons as illustrated in Fig. 1. The aim of this article is to give a series of different examples for both excitation sources in order to illustrate the possibilities of the methods. Discussion of the individual examples is by no means complete, and the reader is referred to the indicated references.

Section 2 briefly describes the experiment. In Section 3, different aspects of angle-scanned XPD are presented with emphasis on the so-called "forward-focusing" regime. Section 4 considers the electronic structure and in particular the Fermi surface (FS) mapping. Conclusions are given in Section 5.

\section{Experimental}

Fig. 1 presents the general idea of the experiment. Two types of photon sources are available, one in the soft X-ray regime and the second in the UV range of energies. They are fixed in space and consist, in the present case, of a dual $\mathrm{Mg} / \mathrm{Si} \mathrm{K} \alpha$ $\mathrm{X}$-ray anode $(h v=1254 \mathrm{eV} / 1740 \mathrm{eV})$ and a $\mathrm{He}$ lamp [He I $(21.2 \mathrm{eV}) / \mathrm{He}$ II $(40.8 \mathrm{eV})]$. The elec- 
trostatic, hemispherical, angle and energy resolving analyzer is also fixed in space, and angle-scanning is performed via motorized sequential sample rotation $[1,2]$. Typically 4000-6000 angular settings are scanned homogeneously distributed over the hemisphere above the sample. Other data collection modes (not discussed here) are possible using different analyzers [3-9]. The important point here is that the experimental procedure is identical either with soft X-rays or with UV photons.

\section{Structure determination}

As shown in Fig. 1, photoelectrons with kinetic energies above $\sim 0.5 \mathrm{keV}$ have scattering properties such that most of the flux is directed along the emitter-scatterer direction, indicating atom-atom directions. Also, this property does not depend strongly on the atomic number. Besides this forward focusing, we find interference fringes due to first-order interference between the direct and the scattered wave giving information on the bond distance [10]. XPD has been reviewed recently for the angle-scanned mode in particular [11] and in general [12-16]. Here, we will focus on a few recent, particularly interesting applications of XPD in the "forward-focusing" regime, but without the quantitative determination of adsorbate positions using $R$-factor analyses discussed elsewhere $[17,18]$.

\subsection{Bulk emission and stereographic projection}

Due to the forward-focusing of photoelectrons, emission along densely packed crystallographic planes appears in the diffraction pattern with enhanced intensity, giving a fingerprint of the crystallographic structure near the surface. In Fig. 2a and 2b, we present the example of an Y-Ni surface alloy that was produced by depositing thin Y films $(\approx 50 \AA)$ on $\mathrm{Ni}(001)$ and $\mathrm{Ni}(110)$ at slightly elevated temperature $(T)\left(T \approx 240^{\circ} \mathrm{C}\right)$ [19]. Note that the data are presented in stereographic projection (SP) and that in a SP, planes become circles. Consulting the phase diagram of $\mathrm{Y}-\mathrm{Ni}$, a wealth of different compounds are found including orthorhombic, monoclinic, hexagonal, tetragonal, trigonal and cubic symmetries. It is now very
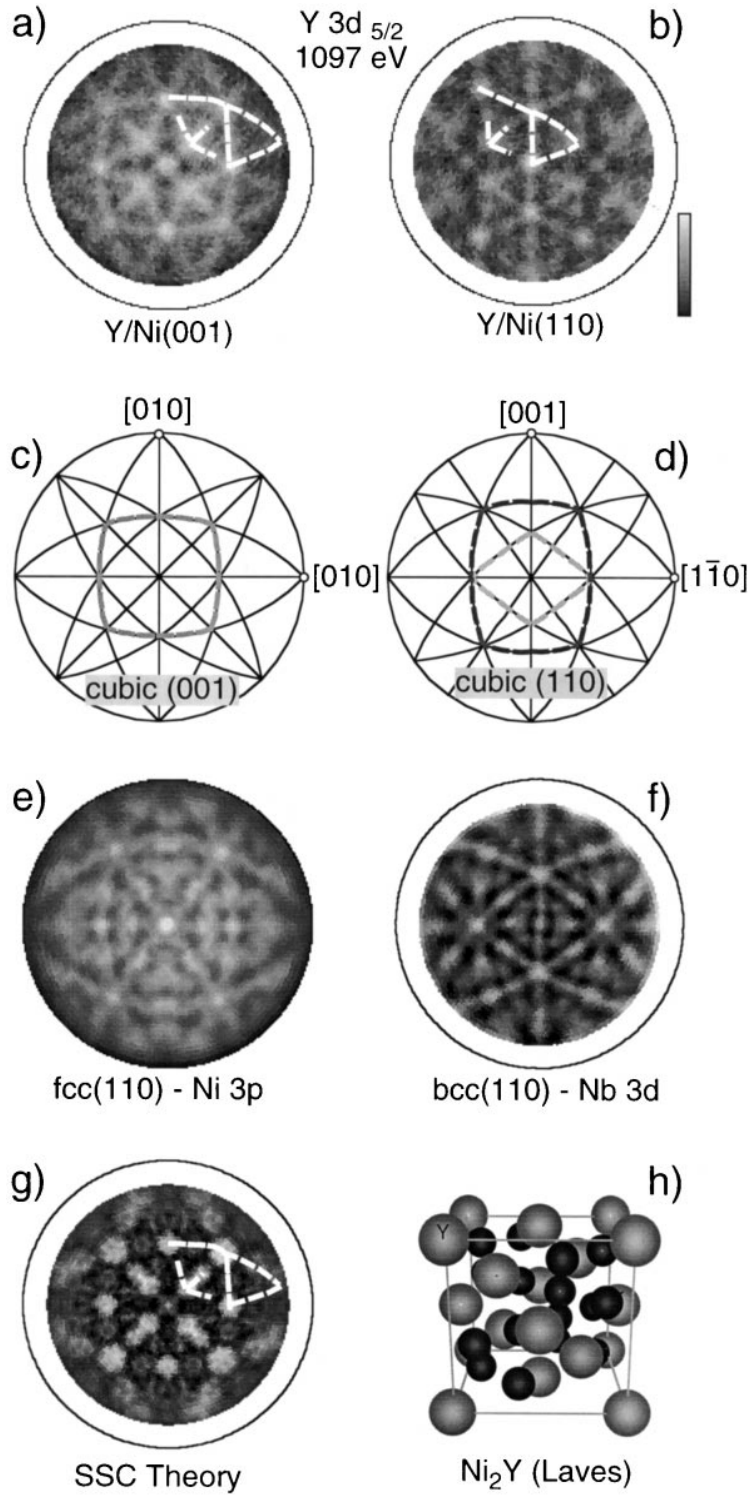

Fig. 2. Stereographic projection (SP) mode of XPD applied to an $\mathrm{Y} / \mathrm{Ni}$ surface alloy: (a) $\mathrm{Y} / \mathrm{Ni}(001)$ deposited at $T \approx 240^{\circ} \mathrm{C}$, experimental $(\mathrm{Mg} \mathrm{K} \alpha)$ Y $3 \mathrm{~d}_{5 / 2}$ photoemission intensity distribution in SP with linear gray-scale representation, plotting high intensities in white; normal emission is located at the center, and $90^{\circ}$ off normal emission is marked by the outer circle; white dashed lines indicate special features; (b) same as (a) but for $\mathrm{Ni}(110)$; (c) SP of the cubic (001) crystallographic structure; (d) same as (c) but for the (110) direction; (e) experiment $(\mathrm{Mg} \mathrm{K} \alpha)$ on a clean fcc(110) surface ( $\mathrm{Ni}(110))$; (f) experiment $(\mathrm{Mg} \mathrm{K} \alpha)$ on a clean bcc(110) surface $(\mathrm{Nb}(110))$; (g) SSC theory for (a); (h) the $\mathrm{Ni}_{2} \mathrm{Y}$ Laves phase proposed for the $\mathrm{Y} / \mathrm{Ni}$ surface alloy with $\mathrm{Y}$ and $\mathrm{Ni}$ as light and dark balls, respectively. 
straightforward to compare SPs of the different crystallographic structures and their high symmetry directions with the measurement (Fig. 2a). Fig. 2c shows the stereographic projection (SP) of high symmetry planes of a cubic crystal structure along the (001) axis. It turns out that the SP (Fig. 2c) of a single domain of the cubic Laves phase of $\mathrm{Ni}_{2} \mathrm{Y}$ (Fig. 2h) matches the experiment (Fig. 2a), allowing us to identify the phase. It is important to notice that not all the planes present in the SP have to occur in the experiment as demonstrated by comparing two cubic phases in Fig. 2e [fcc(110)] and Fig. 2f [bcc(110)]. Clearly, different planes have enhanced intensity as indicated in Fig. $2 \mathrm{~d}$ by thicker, dashed lines. The enhanced intensity depends on the occupation of these planes which is certainly different for fcc and bcc. In Fig. 2g, a model calculation is shown for comparison with Fig. 2a using the single scattering cluster (SSC) scheme [20]. It is well known that absolute intensities are not modeled correctly by SSC calculations. However, all the experimental features are well reproduced by the calculation, as for example, those indicated by the white dashed lines. Furthermore, it is also easy to see that the same crystallographic structure persists on $\mathrm{Ni}(110)$ (compare dashed white lines on Fig. 2a and 2b), the only difference being that it is rotated according to the different orientation of the substrate. The determination of the composition with XPS is compatible with the proposed Laves phase. However, it would not have been possible to determine the phase solely with XPS since many phases have similar compositions lying within the uncertainty of XPS. Furthermore, we notice that XPD could be successfully applied although no low-energy electron diffraction pattern was detected, indicating the absence of any long-range order. Thus, by a simple inspection of SPs, XPD enabled us to identify the alloy phase in question.

\subsection{Bulk emission: fingerprinting}

Another useful mode of XPD is described in Fig. 3a for the example of the Y-Bi2212 $\mathrm{H} T_{\mathrm{c}}$ superconductor. The host material contains $\mathrm{Ca}$, $\mathrm{Sr}, \mathrm{O}, \mathrm{Bi}$ and $\mathrm{Cu}$ and is, in addition, doped with Y. Without studying in detail the rather complicated crystallographic structure, the XPD pattern
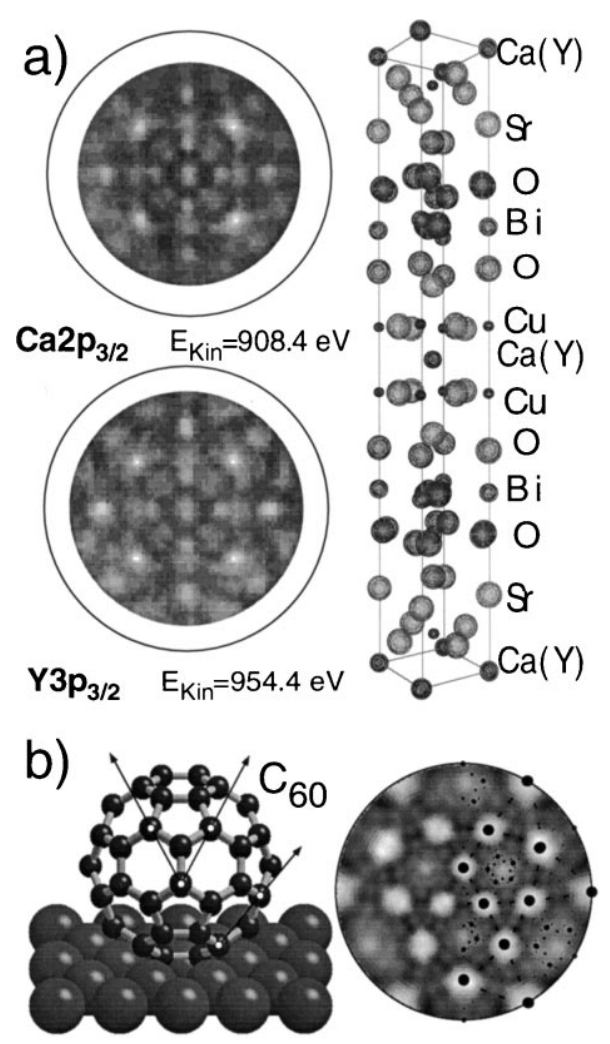

Fig. 3. (a) "Fingerprinting" on the Y-doped Bi-2212 $\mathrm{H} T_{\mathrm{c}}$ superconductor. Since the $\mathrm{Ca}$ and $\mathrm{Y}$ intensity distributions are the same it follows that $\mathrm{Y}$ occupies Ca sites. (b) Molecular orientation of $\mathrm{C}_{60}$ on single crystal surfaces [22]. Because of the forward-focusing property of photoelectrons, the adsorption orientation can be determined (hexagon, pentagon, 6-6 bond, $5-6$ bond or edge atom); on the right hand side, the purely geometrical interpretation of XPD (black spots) is superimposed on to a proper scattering calculation.

of the different host elements can be measured and considered as a characteristic image of the average local real-space environment of the particular element. It appears that the images of the host elements differ distinctly from each other and agree with those measured previously on undoped Bi2212 [21]. Since the Y signal is virtually identical to the $\mathrm{Ca}$ signal, it follows immediately that $\mathrm{Y}$ occupies $\mathrm{Ca}$ sites, at least in a region within the escape depth of the photoelectrons.

\subsection{Molecular orientation}

Since XPD has this wonderful forward-focusing behavior, angular intensity distributions, to a first 
approximation, represent a forward projected image of the atomic structure around the photoemitter. Fig. $3 b$ schematically shows a $\mathrm{C}_{60}$ molecule sitting on a single crystal surface facing it with a hexagon [22]. When irradiated with $\mathrm{Mg} \mathrm{K} \alpha$ radiation, each of the $60 \mathrm{C}$ atoms emits photoelectrons. This is simulated on the right side with an SSC calculation for a single $\mathrm{C}_{60}$ molecule facing the surface with a hexagon. Superimposed on the right side of the calculation are black spots indicating $\mathrm{C}-\mathrm{C}$ interatomic directions, with sizes inversely proportional to the corresponding $\mathrm{C}-\mathrm{C}$ distances. This comparison shows how well the purely geometrical interpretation works in this case. Several possibilities with high symmetry exist for a $\mathrm{C}_{60}$ molecule to face the surface, namely with a hexagon, a pentagon, two atoms between two hexagons (6-6 bond) or between a hexagon and a pentagon (6-5 bond), and finally with a single $\mathrm{C}$ atom forming the edge between two hexagons and a pentagon. It is straightforward to compare experiments to SSC calculations for differently oriented molecules. Experiments allow to show unambiguously and directly that $\mathrm{C}_{60}$ faces the $\mathrm{Cu}(111)$ and the $\mathrm{Al}(111)$ surfaces with the hexagon, the $\mathrm{Cu}(110)$ surface with a $6-5$ bond and $\mathrm{Al}(001)$ with an edge atom. For more details, see Ref. [22].

\section{Fermi surface mapping}

Intrinsically angle-resolved UV photoelectron spectroscopy (ARUPS) is suited best for twodimensional (2D) electron systems, i.e. where the perpendicular component of the wave vector $\left(\boldsymbol{k}_{\perp}\right)$ does not play any role. This is the case because in ARUPS, the parallel component of the wave vector $\left(\boldsymbol{k}_{\|}\right)$of the photoelectron is conserved but not $\boldsymbol{k}_{\perp}$. Furthermore, attractive measuring modes made it possible to map the FS of $2 \mathrm{D}$ systems directly [23,24]. Nevertheless, one wishes to gain information on 3D FSs as well. Very promising results have been obtained for $\mathrm{Cu}$ [25] and $\mathrm{Ni}$ [26]. The dilemma of visualizing bulk states in general is indicated in Fig. 4: For every particular $\boldsymbol{k}$ vector, many different energies may be a solution to Schrödinger's equation. Therefore, either one chooses only few $\boldsymbol{k}$ vectors, preferentially along high symmetry directions within the BZ as in Fig. 4a [27] to display all energies, or one chooses one single energy, e.g. the Fermi energy $\left(E_{\mathrm{F}}\right)$, and displays all corresponding $\boldsymbol{k}$ vectors ending up with a constant energy surface, e.g. the FS, as in Fig. 4b [28]. The analogy between $E(\boldsymbol{k})$ and $\boldsymbol{k}(E)$ is seen by following a path from the L-point to the $\mathrm{W}$-point in $\mathrm{k}$-space. Very shortly after $\mathrm{L}$, the FS is crossed. This is also evident from the $E(\boldsymbol{k})$ in Fig. 4a. From the experimental point of view, the situation is very much the same in that either energy spectra can be measured for a few angles (Fig. 4c) or; intensities for example at $E_{\mathrm{F}}$, for all the angles. This is displayed in Fig. $4 \mathrm{~d}$ where intensities from $E_{\mathrm{F}}$ are plotted using a linear gray scale with high intensities in black. The data are plotted linearly in $\boldsymbol{k}_{\|}$(via $\left|\boldsymbol{k}_{\|}\right|=k_{0} \sin \theta, k_{0}$ the absolute value of the final state $\boldsymbol{k}$-vector in vacuum) to compare directly with theory in Fig. 4e. Normal emission occurs at the center of the image and grazing emission towards the outside. Intensities collected at $E_{\mathrm{F}}$ along the azimuthal scans taken at a polar angle of $66^{\circ}$ off normal in Fig. 4c are indicated with an arrow in the Fermi scan of Fig. 4d. Assuming a free electron model for the final state, we obtain a high intensity in our energy window centered at $E_{\mathrm{F}}$ when the free electron final state (FEFS) sphere intersects the FS in the extended zone scheme, i.e. conditions for energy and momentum conservation are fulfilled. This is illustrated in Fig. 4f for a plane containing the (001), (111) and (110) directions. Final-state spheres for different photon energies (He I, He II) appear as circles with different radii. As a matter of fact, using tunable synchrotron radiation, it should be possible to slice the FS piece by piece. In Fig. 4e, the drawing plane of Fig. $4 \mathrm{f}$ is represented by the dashed line entering the plot from the upper side through the "neck" and ending up in the "bone" [25]. The dotted lines mark all the intersecting points of the He I FEFS sphere with the calculated FS in the extended zone scheme, analogous to what is done for a single plane in Fig. 4f. The theory in Fig. 4e fits very well with the experiment in Fig. 4d, except for the small ring in the center representing the well-known surface state of the $\mathrm{Cu}(111)$ surface and which, of course, is not reproduced in a bulk calculation. The white 
a)

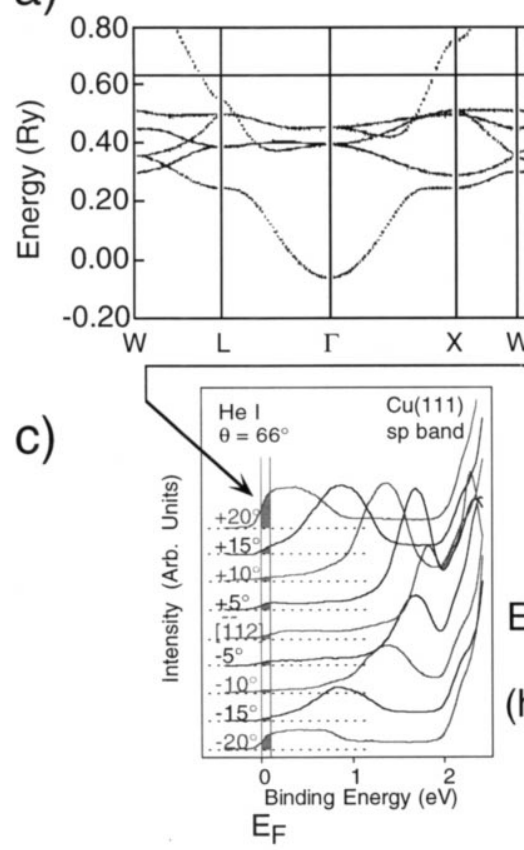

c)
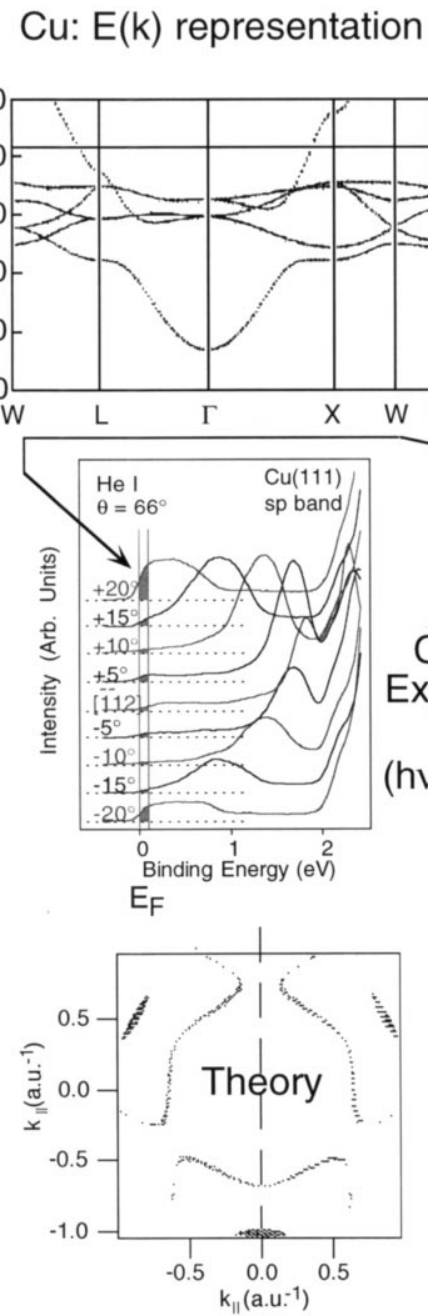

g)

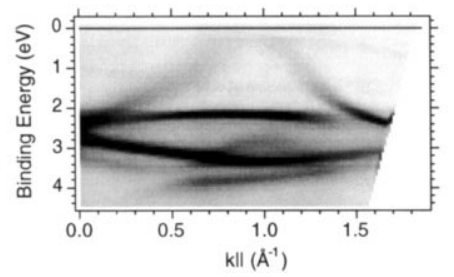

b)

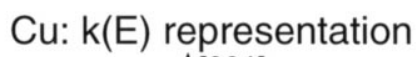

Cu: $k(E)$ representation

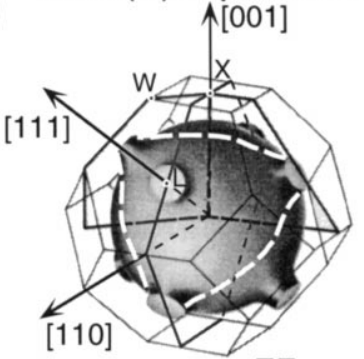

$[\overline{1} \overline{1} 2]$

d)

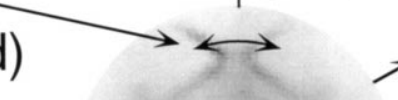

[2111]

$\mathrm{Cu}(111)$

Experiment

$\mathrm{Hel}$

$(\mathrm{hv}=21.2 \mathrm{eV})$

f)

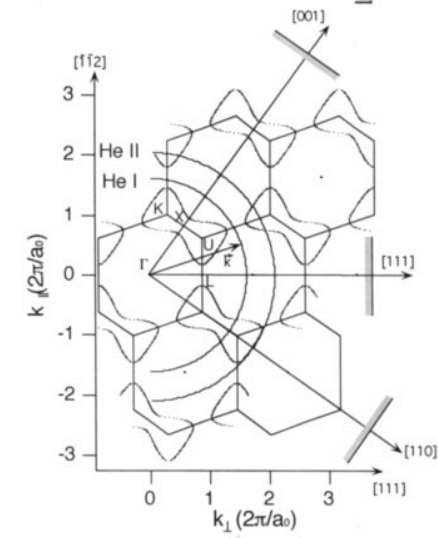

h)

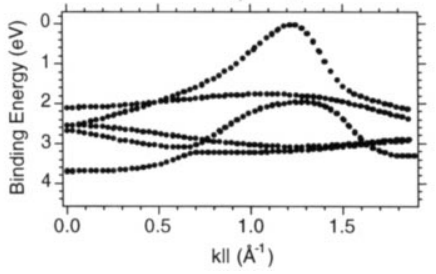

Fig. 4. Angle-scanned UV photoemission for Fermi surface (FS) and band mapping: (a) $E(\boldsymbol{k})$ representation of the bandstructure [27]; (b) $\boldsymbol{k}\left(E_{\mathrm{F}}\right), \mathrm{FS}$ of $\mathrm{Cu}$ [28]; (c) He I excited EDCs from $\mathrm{Cu}(111)$ at a polar angle of $66^{\circ}$; azimuthal angles around the [1112] direction are indicated; vertical lines indicate the energy window used in the angle scans of (d); (d) $E_{\mathrm{F}} \operatorname{scan}$ on $\mathrm{Cu}(111)$, i.e. the total intensity of He I excited photoelectrons collected in the energy window sketched in (c), measured as a function of $\boldsymbol{k}_{\|}$; normal emission is located in the center; the gray-scale is linear with high intensities in black; (e) cuts through the bulk FS using a FEFS (see text); (f) high symmetry plane perpendicular to the [110] direction; the solid line polygons correspond to the cut through the bulk BZ; partly dotted, a calculation of the FS; the typical necks at the L-point and bones around X are evident; the half circles around the $\Gamma$ point represent the FEFS wave vector for the He I and II photon energies; (g) "carpet", i.e. EDCs continuously taken as a function of polar angle on $\mathrm{Cu}(001)$ and interpolated in $\boldsymbol{k}_{\|}$, for band mapping; (h) corresponding calculation (see text). 
dashed line in Fig. 4b indicates the measured spherical cut across the bulk FS. Other groups have measured $E_{\mathrm{F}}$ scans on $\mathrm{Cu}$ as well [29-31].

Yet another mode of angle-scanned UV photoemission is indicated in Fig. $4 \mathrm{~g}$ and $4 \mathrm{~h}$. A large set of complete EDCs is taken along a high symmetry azimuth and assembled into a so-called "carpet". The data are taken as a function of angle and then mapped on to a regular $\boldsymbol{k}_{\|}$grid. Here, $\boldsymbol{k}_{\|}=0$ corresponds to the $\mathrm{Cu}(001)$ normal emission direction and increases towards the (111) direction, i.e. the measurements follow the He I FEFS circle in Fig. 4f. At approximately $k_{\|}=1 \AA^{-1}$, the sp band is seen to cross the Fermi level towards empty states and to move down again. This corresponds to the intersection of the neck and the bulk BZ in Fig. 4f. For the calculation shown in Fig. 4h, again, a FEFS has been assumed. For different binding energies, the radii of the circles representing the FEFS have been chosen accordingly. A point is then plotted in the case of energy and momentum conservation [32]. We find all experimental bands in the calculation. However, agreement of the relative positions might be better. A possible explanation is that the FEFS is not a good enough approximation. Depending on the slope of the initial state constant energy surfaces, a slight deviation from a sphere in the final state results already in a considerable $\boldsymbol{k}$ displacement of the crossings and therefore in shifts in Fig. 4h. Nevertheless, in such a manner, extended band mapping is possible.

As a second example, showing $T$-dependent experiments, we discuss $\mathrm{Ni}$, a magnetic material. Analogous to the case of $\mathrm{Cu}$, the intensity of photoelectrons from $E_{\mathrm{F}}$ has been mapped over much of the hemisphere [26]. Fig. 5 displays $E_{\mathrm{F}}$ maps as a function of $T$ for $T \mathrm{~s}$ below and above the Curie temperature $T_{\mathrm{C}}$ together with a spinresolved calculation where spin up and down regions are marked in light and dark gray, respectively. On the right side, a series of measurements covering one-quarter of the azimuth is presented, as collected with increasing $T$, visualizing the development while going from $T<T_{\mathrm{C}}$ to $T>T_{\mathrm{C}}$. Because of the good agreement of theory and room temperature (RT) experiment, up and down spins can be readily identified without any explicit spin detection and sample magnetization. Regions marked
$\mathrm{A}, \mathrm{B}$ and $\mathrm{C}$ all show moving bands as $T$ is increased. Some spectral weight around $\mathrm{A}^{\prime}$, however, seems to remain in place as opposed to what is expected for a Stoner behavior of magnetism. The region of $\mathrm{A}^{\prime}$ consists of overlapping up and down bands as can be seen from the calculation. Recent measurements and calculations analogous to those presented above identified the character of the moving and remaining bands [33]. It turns out that the bands staying in place are up and down sp-bands, so steeply dispersing in energy that they are not resolved in angle in the experiment. Because of the large slope, sp-bands moving up respectively down in energy while collapsing will not result in any observable movement in $\mathrm{k}$-space. Therefore, the observation of these bands staying in place as we go across $T_{\mathrm{C}}$ does not oppose a Stoner-like behavior. For a detailed discussion of the experiments on $\mathrm{Ni}$ and implications on models of the magnetism, see Refs. $[26,33]$.

\section{Conclusions}

A survey has been given on angle-scanned photoemission. We have shown that by switching the excitation source between soft X-rays and UV photons, within the same experiment, both, the geometrical and electronic structure can be studied.

In particular, due to the forward-focusing properties together with a full hemispherical gray-scale mapping of photoelectron intensities in a SP, the experiment can be compared with the SP of a proposed crystallographic structure to accept or dismiss it. The measurement can also be viewed as a fingerprint of the local real-space environment of a specific element. XPD also proved very useful for determining very uniquely the orientation of adsorbed $\mathrm{C}_{60}$ molecules.

In the UV regime, the method is capable of measuring sections across the bulk FS, to perform band mapping, and even to gain spin-resolved information. Assuming a FEFS model, a good agreement between calculations and experiments can be achieved. Nevertheless, deviations from a FEFS may introduce distortions and shifts in the calculations. 

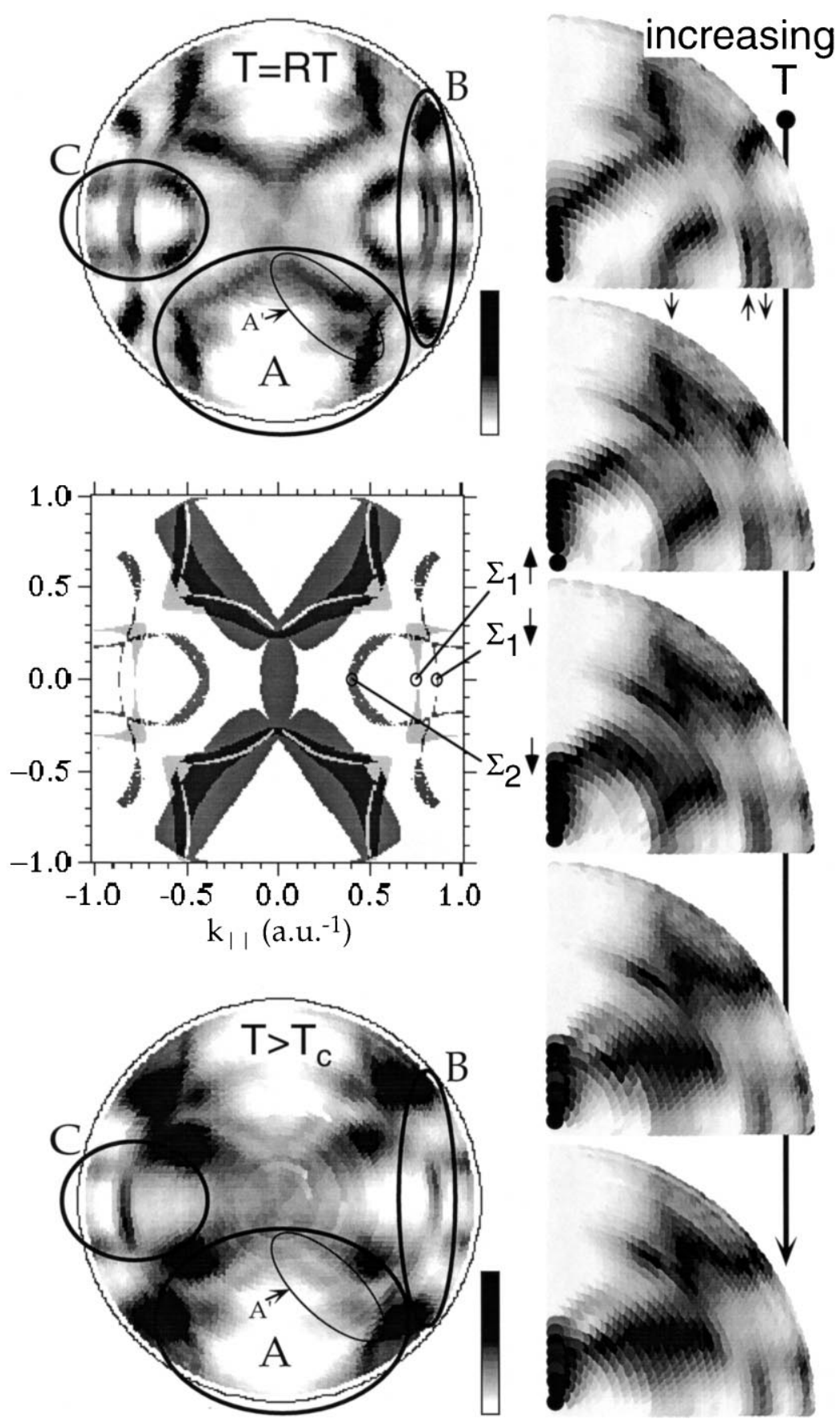

Fig. 5. Experimental $E_{\mathrm{F}}$ scans on $\mathrm{Ni}(110)$ as a function of $T$ together with a spin-resolved calculation [26] (left side, center). A linear gray-scale is applied with high intensities in black. For the experiments with increasing $T$, only one-quarter of the azimuthal range is measured and a maximal contrast has been chosen for every polar angle. The complete measurements at $\mathrm{RT}$ and above $T_{\mathrm{c}}$ have been normalized to a smooth polar angle dependent background for better visualization. The scaling is linear in $\boldsymbol{k}_{\|}$, and normal emission is situated in the center (corner) of the circle (quarter circle) which represents grazing emission of photoelectrons. A, B, C and $\mathrm{A}^{\prime}$ mark regions of interest. 
In the future, the method might prove most useful for synthetically prepared adsorbate systems where both the geometric and electronic structure are unknown [17,34]. Its value might even be enhanced when combined with state-of-the-art calculations [35].

\section{Acknowledgements}

Skilful technical assistance was provided by $\mathrm{E}$. Mooser, O. Raetzo, Ch. Neururer and F. Bourqui. This Project has been supported by the Fonds National Suisse de la Recherche Scientifique.

\section{References}

[1] J. Osterwalder, T. Greber, A. Stuck, L. Schlapbach, Phys. Rev. B 44 (1991) 13764.

[2] D. Naumović, A. Stuck, T. Greber, J. Osterwalder, L. Schlapbach, Phys. Rev. B 47 (1993) 7462.

[3] D.G. Frank, T. Golden, O.M.R. Chyan, A.T. Hubbard, J. Vac. Sci. Technol. A 9 (1991) 158.

[4] H.A. Engelhardt, W. Bäck, D. Menzel, H. Liebl, Rev. Sci. Instrum. 52 (1981) 835.

[5] R.C.G. Leckey, J.D. Riley, Appl. Surf. Sci. 22/23 (1985) 196.

[6] S. Kono, N. Nakamura, K. Anno, S. Teruyama, Surf. Sci. 271 (1992) 596.

[7] D.E. Eastman, J.J. Donelon, C. Hien, F.J. Himpsel, Nucl. Instrum. Meth. 172 (1980) 327.

[8] D. Rieger, R.D. Schnell, W. Steinmann, V. Saile, Nucl. Instrum. Meth. 208 (1983) 777.

[9] H. Daimon, S. Ino, Rev. Sci. Instrum. 61 (1990) 57.

[10] R. Fasel, P. Aebi, J. Osterwalder, L. Schlapbach, Surf. Sci. 331332333 (1995) 80.

[11] J. Osterwalder, P. Aebi, R. Fasel, D. Naumović, P. Schwaller, T.J. Kreutz, L. Schlapbach, T. Abukawa, S. Kono, Surf. Sci. 331332333 (1995) 1002.

[12] C.S. Fadley, in: R.Z. Bachran (Ed.), Synchrotron Radia- tion Research: Advances in Surface Science, Plenum, New York, 1990, Chap. 11.

[13] W.F. Egelhoff, Jr, Crit. Rev. Solid State Mater. Sci. 16 (1990) 213.

[14] S.A. Chambers, Surf. Sci. Rep. 16 (1992) 261.

[15] H.P. Bonzel, Progr. Surf. Sci. 42 (1993) 219.

[16] C.S. Fadley, Surf. Sci. Rep. 19 (1993) 231.

[17] R. Fasel, P. Aebi, J. Osterwalder, L. Schlapbach, R.G. Agostino, G. Chiarello, Phys. Rev. B 50 (1994) 14516.

[18] J.A. Martín-Gago, R. Fasel, J. Hayoz, R.G. Agostino, D. Naumović, P. Aebi, L. Schlapbach, Phys. Rev. B 55 (1997) 12896.

[19] D. Naumović et al., to be published.

[20] D.J. Friedman, C.S. Fadley, J. Electron Spectrosc. Relat. Phenom. 51 (1990) 689.

[21] J. Osterwalder, P. Aebi, P. Schwaller, L. Schlapbach, M. Shimoda, T. Mochiku, K. Kadowaki, Appl. Phys. A 60 (1994) 247.

[22] R. Fasel, P. Aebi, R.G. Agostino, J. Osterwalder, A. Santaniello, L. Schlapbach, Phys. Rev. Lett. 76 (1996) 4733.

[23] A. Santoni, L.J. Terminello, F.J. Himpsel, T. Takahashi, Appl. Phys. A 52 (1991) 229.

[24] P. Aebi, J. Osterwalder, P. Schwaller, L. Schlapbach, M. Shimoda, T. Mochiku, K. Kadowaki, Phys. Rev. Lett. 72 (1994) 2757.

[25] P. Aebi, J. Osterwalder, R. Fasel, D. Naumović, L. Schlapbach, Surf. Sci. 307308309 (1994) 917.

[26] P. Aebi, T.J. Kreutz, J. Osterwalder, R. Fasel, P. Schwaller, L. Schlapbach, Phys. Rev. Lett. 76 (1996) 1150.

[27] V.L Moruzzi, J.F. Janak, A.R. Williams, Calculated Electronic Properties of Metals, Pergamon, New York, 1978.

[28] N.W. Ashcroft, N.D. Mermin, Solid State Physics, Saunders College, Philadelphia, PA.

[29] A.P.J. Stampfl, J.A. Con Foo, R.C.G. Leckey, J.D. Leckey, J.D. Riley, R. Denecke, L. Ley, Surf. Sci. 331332333 (1995) 1272.

[30] J. Avila, C. Casado, M.C. Asensio, J.L. Perez, M.C. Muñoz, F. Soria, J. Vac. Sci. Technol. A 13 (1995) 1501.

[31] Z. Qu, A. Goonewardene, K. Subramanian, J. Karunamuni, N. Mainkar, L. Ye, R.L. Stockbauer, R.L. Kurtz, Surf. Sci. 324 (1995) 133.

[32] M. Bovet, Diploma thesis, Université de Fribourg, 1997.

[33] T.J Kreutz, PhD thesis, Universität Zürich, 1997.

[34] R. Fasel, P. Aebi, R.G. Agostino, J. Osterwalder, L. Schlapbach, Phys. Rev. B 54 (1996) 5893.

[35] C. Stampfl, K. Kambe, R. Fasel, P. Aebi, M. Scheffler, in preparation. 